\title{
High harmonic generation in gas-filled photonic crystal fibers
}

\author{
Florian Wiegandt ${ }^{1}$, Patrick N. Anderson ${ }^{1}$, Fei Y ${ }^{2}$, Daniel J. Treacher ${ }^{1}$, David T. Lloyd ${ }^{1}$, \\ Peter J. Mosley', Simon M. Hooker', Ian A. Walmsley ${ }^{I}$ \\ ${ }^{l}$ Clarendon Laboratory, University of Oxford, Parks Road, Oxford, OX1 3PU, UK \\ ${ }^{2}$ Centre for Photonics and Photonic Materials, Department of Physics, University of Bath, Bath, BA2 7AY, UK
}

High harmonic generation (HHG) is a promising tabletop source of coherent short wavelength radiation, with applications spanning science and engineering [1]. However, the low conversion efficiency and low average power of conventional few-kHz near-infrared (NIR) driving lasers limits the photon flux of such sources. Scaling this technique to $\mathrm{MHz}$ driving lasers requires strong focusing due to the limited pulse energy, and as a result the interaction volume is greatly reduced. It has been shown that this may be mitigated by restricting HHG to a photonic crystal fiber (PCF) $[2,3]$. Here, we explore HHG in the latest generation of negative curvature PCFs [4] and achieve the highest photon energies to date.

Our experiment is shown in Fig. 1b. Here, 30 fs pulses from a high energy Ti:Sa laser system, operating at a $1 \mathrm{kHz}$ repetition rate are attenuated, spatially filtered and launched into a $10.5 \mathrm{~mm}$ long section of a PCF with a $22.5 \mu \mathrm{m}$ core diameter. The PCF is mounted between two chambers. The first of these is filled with 800 mbar of gas, and high-vacuum is maintained throughout the second. The extreme ultraviolet (XUV) output of the PCF is analyzed using a flat field XUV spectrometer. Additionally, a fiber coupled NIR spectrometer is used to characterize the residual driving laser. In argon we observe HHG at launched pulse energies of approximately $5 \mu \mathrm{J}$. Using neon this threshold shifts to $10 \mu \mathrm{J}$, and increasing the pulse energy to $15 \mu \mathrm{J}$ yields harmonics with energies up to $66 \mathrm{eV}$ ( $43^{\text {rd }}$ harmonic order, Fig. 1a). These are, to our knowledge the highest photon energies recorded from a PCF to date. In addition, we have observed ionization-induced reshaping of the driving laser as it passes through the PCF. The fundamental spectrum of the driving laser at the output of an argon-filled PCF is shown in Fig. 1d for a range of pulse energies. When the XUV output is analyzed under identical conditions (Fig. 1c) this behavior is shown to map onto the harmonic spectrum, towards a continuum like regime. We believe this mechanism may lead to the realization of compact, high average power sources of XUV supercontinuum.
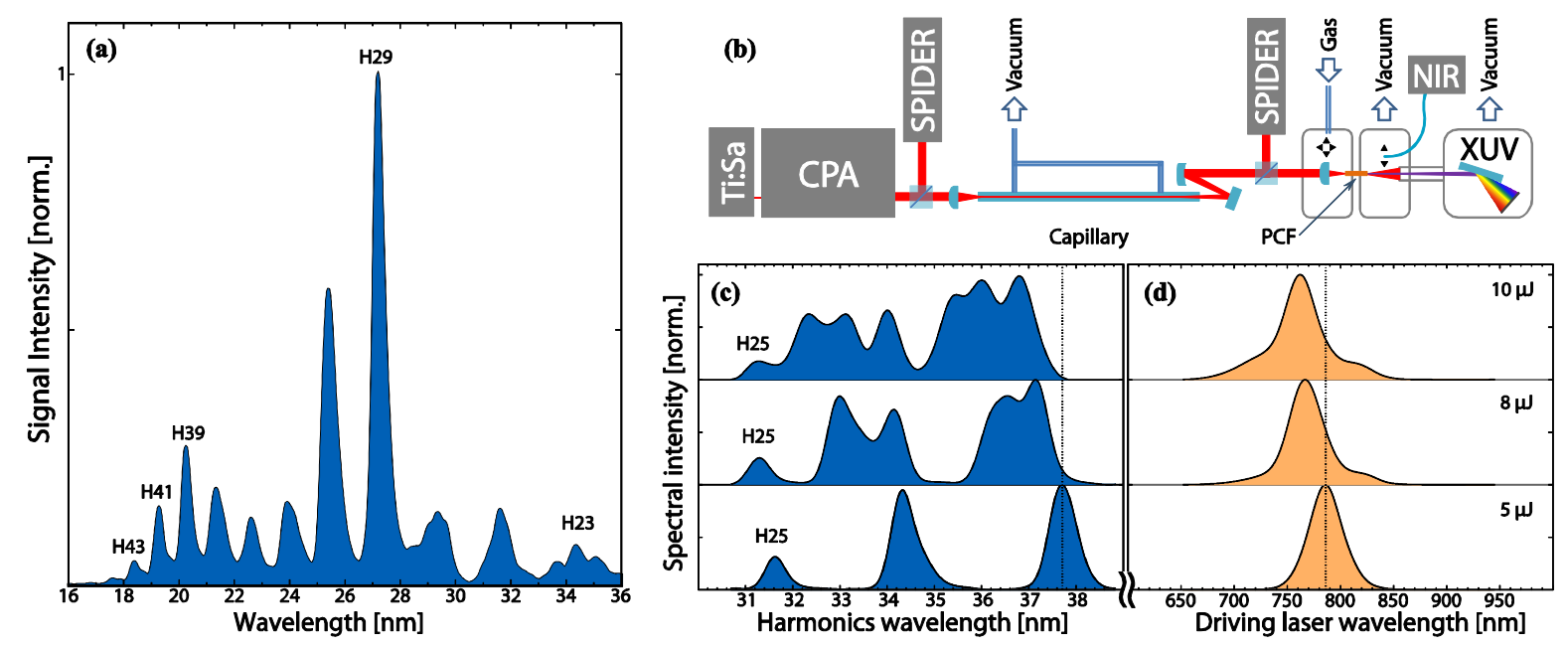

Fig. 1. (a) Harmonic spectrum from a neon-filled PCF driven by $15 \mu \mathrm{J}$ pulses. (b) Optical setup. Ti:Sa oscillator; CPA, chirped pulse amplifier; SPIDER, spectral phase interferometry for direct electric-field reconstruction; PCF, photonic crystal fiber; NIR, near-infrared spectrometer; XUV, extreme ultraviolet spectrometer. (c) Harmonic spectrum from an argon-filled $\mathrm{PCF}$ at a range of pulse energies. (d) The corresponding fundamental spectrum.

\section{References}

[1] E. R. Shanblatt, C. L. Porter, D. F. Gardner, G. F. Mancini, R. M. Karl Jr., M. D. Tanksalvala, C. S. Bevis, V. H. Vartanian, H. C. Kapteyn, D. E. Adams, and M. M. Murnane, "Quantitative Chemically-Specific Coherent Diffractive Imaging of Buried Interfaces using a Tabletop EUV Nanoscope" Nano Lett., 16, 5444 (2016)

[2] H. Ren, A. Nazarkin, J. Nold, P.S.J. Russell, "Quasi-phase-matched high harmonic generation in hollow core photonic crystal fibers" Opt. Express, 16, 17052 (2008).

[3] O. H. Heckl, C. R. E. Baer, C. Kränkel, S. V. Marchese, F. Schapper, M. Holler, T. Südmeyer, J. S. Robinson, J. W. G. Tisch, F. Couny, P. Light, F. Benabid and U. Keller, "High harmonic generation in a gas-filled hollow-core photonic crystal fiber" Appl. Phys. B, 97, 369 (2009).

[4] F. Yu and J. C. Knight, "Spectral attenuation limits of silica hollow core negative curvature fiber" Opt. Express 21, 21466-21471 (2013). 